\title{
Avaliação do grau de orientação de fibras de aço induzida na moldagem de elementos planos com concreto autoadensável
}

\author{
Evaluation of steel fiber orientation in \\ slab elements induced during casting \\ with self-compacting concrete
}

Ricardo dos Santos Alferes Filho ${ }^{1}$, Renata Monte ${ }^{1}$, Antonio Domingues de Figueiredo ${ }^{1}$

\footnotetext{
${ }^{1}$ Escola Politécnica da Universidade de São Paulo - Caixa Postal 61548. CEP 05508-900, São Paulo, SP, Brasil. e-mail: alferes.ricardo@gmail.com, antonio.figueiredo@usp.br, renata.monte@usp.br
}

\section{RESUMO}

O uso de concreto autoadensável (CAA) aliado ao concreto reforçado com fibras (CRF) tem sido objeto de várias pesquisas recentes. A combinação da fluidez do CAA com o uso de fibras aumenta a probabilidade de que, no processo de lançamento, seja possível causar orientação preferencial nas fibras, inclusive em elementos planos. Isto pode ser benéfico ou prejudicial ao desempenho estrutural, uma vez que a capacidade resistente pós-fissuração do CRF depende fortemente da disposição e orientação das fibras. Devido a isso, é necessário o desenvolvimento de metodologia que possibilite avaliar esta condição. O uso de ensaios especiais, como o indutivo, pode ser uma ferramenta de controle que auxilie na compreensão da influência das condições de moldagem na orientação das fibras. No entanto, não há muitas pesquisas voltadas à parametrização desta condição em elementos planos. Este estudo traz como objetivo a demonstração da efetividade de utilização do ensaio indutivo em testemunhos cúbicos extraídos de uma placa moldada com concreto autoadensável reforçado com fibras de aço. Como resultado, o ensaio indutivo permitiu medir a variação na concentração de fibras em cada testemunho e, através da orientação de fibras medida em cada cubo, traçar linhas de fluxo que apontem a influência da moldagem na orientação preferencial das fibras.

Palavras-chave: Orientação de fibras. Ensaio indutivo. Elementos planos. Concreto autoadensável reforçado com fibras.

\section{ABSTRACT}

The use of self-compacting concrete (SCC) combined with fiber reinforced concrete (FRC) has been the subject of several recent studies. The combination of the fluidity of the SCC with the use of fiber increases the possibility that, during the casting process, there is preferred orientation of the fibers, even in the case of slab elements. This could provoke an enhancement or a loss in structural performance, once the post cracking strength of FRC relies on the positioning and orientation of the fibers. Because of this, the development of a methodology is needed in order to allow the assessment of this condition. The use of special test methods, as the inductive test, may be a useful tool to help to understand the influence of the casting conditions in fiber orientation. However, there are not many studies focused on the parameterization of this condition in slab elements. The present study aims to use the inductive test method in cubic cores sawn from a slab made of steel fiber reinforced self-compacting concrete. As result, the inductive method was able to assess the variation in the fiber concentration and the fiber orientation in each core. The use of the individual cubes measured results turn possible to draw flow lines that point to the influence of the casting in the preferred fiber orientation and concentration.

Keywords: Fiber orientation. Inductive method. Slabs. Fiber reinforced self-compacting concrete. 


\section{INTRODUÇÃO}

A utilização do concreto reforçado com fibras para finalidades estruturais vem sendo ampliada recentemente, especialmente após a publicação do novo código modelo da FIB [1]. Di PRISCO et al.[2] chamaram a atenção para o fato que a principal contribuição da fibra para o comportamento de elementos estruturais ocorre na etapa pós-fissuração. Assim, a resistência residual de elementos estruturais irá depender das condições de reforço oferecidas pelas fibras que podem ser afetadas por uma série de fatores como o seu teor, resistência e geometria [3].

A disposição das fibras no concreto é outro fator de grande importância na definição de seu comportamento mecânico. A orientação das fibras e sua concentração podem mudar completamente a resposta dos elementos estruturais frente aos esforços aplicados. Se bem explorada, pode ser responsável pela otimização do desempenho mecânico e da tenacidade, mas, por outro lado, caso a orientação seja inapropriada, há a possibilidade de que vários efeitos indesejáveis à estrutura sejam causados [4].

Devido à fluidez elevada, o concreto autoadensável possibilita que esta orientação das fibras seja feita durante o processo de lançamento [4-7], o que torna a etapa de produção um evento de destacada importância, visto que as condições de moldagem poderiam então ser responsáveis por comprometer o desempenho mecânico de um elemento estrutural.

$\mathrm{O}$ aumento do interesse por este tema, tem feito com que pesquisas recentes foquem a análise no efeito da moldagem sobre o comportamento pós-fissuração de elementos planos, nos quais a maior influência atuante na orientação das fibras é dada pelo fluxo, uma vez que o efeito parede é menos presente [8,9]. Logo, é necessário o contínuo desenvolvimento de estudos que abordem os efeitos das condições de moldagem, que, ao ter possível efeito na concentração e orientação das fibras, pode influenciar no comportamento mecânico de elementos estruturais moldados com concreto autoadensável reforçado com fibras de aço.

Outros estudos têm buscado usar das propriedades elétricas das fibras de aço em seus métodos de ensaio para análise da orientação de fibras em elementos planos de concreto. LATASTE et al. [10] mediram variação na resistividade elétrica em várias posições de lajes moldadas com concreto reforçado com fibras de aço. AL-MATTARNEH [11], por sua vez, mediu variações na impedância, também buscando informações sobre orientação de fibra de aço em lajes de concreto. No entanto, há outras possibilidades atualmente para verificação simultânea do teor e da orientação das fibras. Neste sentido, o ensaio indutivo é bastante promissor [12-13] tendo sido utilizado para parametrizar o concreto projetado onde a orientação preferencial é marcante e o teor é variável em função do processo de reflexão [14]. Assim, optou-se aqui por avaliar a aplicabilidade deste método para parametrizar a orientação e concentração das fibras em elementos planos. Este estudo então busca utilizar o método indutivo em testemunhos extraídos, como forma de medida da concentração e orientação de fibras de aço em elementos planos moldados com concreto autoadensável de modo a tornar possível mapear a distribuição e orientação das fibras nestes elementos.

\section{MATERIAIS E MÉTODOS}

O programa experimental foi fundamentado na moldagem de uma placa de concreto reforçado com fibras, cujo ponto de lançamento de concreto foi fixado no centro da forma, a aproximadamente $20 \mathrm{~cm}$ do fundo. Como foi utilizado um concreto autoadensável, não houve qualquer interferência na condição de compactação e preenchimento do molde que não fosse o próprio fluxo de concreto. Assim, as fibras foram orientadas apenas pelo fluxo do concreto. Após a moldagem e cura da placa foram extraídos testemunhos a partir do corte de cubos, submetidos ao ensaio indutivo para avaliação da orientação das fibras de aço presentes em cada cubo.

\subsection{Materiais e traço}

No estudo experimental foi utilizada uma matriz de concreto e adicionado um teor de $20 \mathrm{~kg} / \mathrm{m}^{3}$ da fibra de aço Dramix 65/35-BG que está demonstrada na Figura 1. Esta fibra é fornecida colada e com ancoragem nas pontas, e apresenta as propriedades descritas na Tabela 1, segundo informações fornecidas pelo fabricante. Os materiais que compunham a matriz de concreto bem como o traço utilizado foi obtido a partir de uma usina de concreto com operação regular na cidade de São Paulo. As proporções dos materiais utilizados, bem como a sua tipificação, estão indicadas na Tabela 2. Nessa mesma tabela é apresentado consumo de cada material e, também, a massa específica do concreto. A Figura 2 apresenta a distribuição granulométrica do cimento e dos agregados.

A mistura foi fornecida diretamente pela concreteira através de um caminhão betoneira com uma car- 
ga de quatro metros cúbicos. Com isso, garantiu-se a exata reprodução das condições de fornecimento prático do material. As fibras foram adicionadas quando da chegada do caminhão ao laboratório da Universidade de São Paulo. Neste momento também foi adicionado o aditivo superplastificante. Utilizou-se o aditivo GRACE ADVA 525, baseado em policarboxilato para garantir as condições de fluidez e autoadensabilidade no concreto.

Tabela 1: Características da fibra utilizada segundo informações fornecidas pelo fabricante.

\begin{tabular}{l|c|c}
\hline PROPRIEDADE & VALOR & UNIDADE \\
\hline Resistência à tração & 1345 & $\mathrm{MPa}$ \\
\hline Módulo de Young & 210000 & $\mathrm{MPa}$ \\
\hline Comprimento (l) & 35 & $\mathrm{~mm}$ \\
\hline Diâmetro (d) & 0,55 & $\mathrm{~mm}$ \\
\hline Fator de forma (1/d) & 65 & - \\
\hline
\end{tabular}

Figura 1: Fibra utilizada (foto própria)

Tabela 2: Traço (em massa) do concreto, consumo dos materiais e massa específica do concreto.

\begin{tabular}{l|c|c}
\hline MATERIAL & TRAÇO & CONSUMO $\left(\mathbf{k g} / \mathbf{m}^{3}\right)$ \\
\hline Cimento CPII E 40 & 1,00 & 404 \\
\hline Areia artificial & 1,01 & 410 \\
\hline Areia de quartzo & 1,41 & 569 \\
\hline Brita 0 $\left(\phi_{\text {máx. }}=9,5 \mathrm{~mm}\right)$ & 2,14 & 864 \\
\hline Fibra de aço 65/35 & 0,05 & 20 \\
\hline Água & 0,45 & 182 \\
\hline Aditivo polifuncional & $0,56 \%$ & 2 \\
\hline Aditivo superplastificante & $1,13 \%$ & 5 \\
\hline Massa específica & \multicolumn{2}{|c}{$2455 \mathrm{~kg} / \mathrm{m}^{3}$} \\
\hline Espalhamento & \multicolumn{2}{|c}{$2 \mathrm{~s}$} \\
\hline$T_{500}$ & $\mathrm{~mm}$ \\
\hline
\end{tabular}




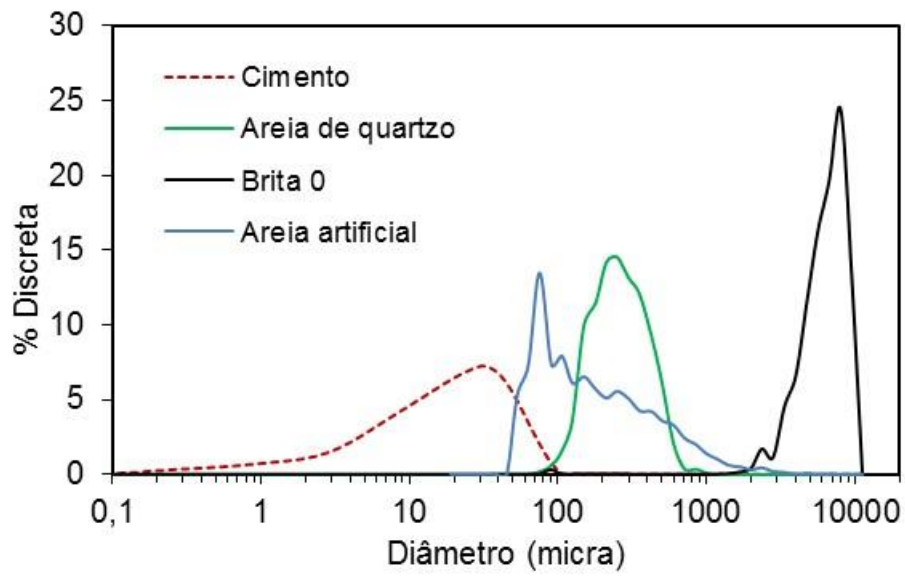

Figura 2: Distribuição granulométrica dos materiais.

\subsection{Moldagem e corte da placa}

Imediatamente antes da moldagem da placa, uma amostra do concreto foi retirada para a caracterização das suas propriedades no estado fresco. Assim, foram feitos os ensaios de espalhamento - slump flow - e tempo de escoamento $\mathrm{T}_{500}$, segundo a metodologia proposta pela EFNARC [15]. Foi moldada uma placa quadrada, com $60 \mathrm{~cm}$ de lado e $10 \mathrm{~cm}$ de altura especificamente para este estudo. O lançamento do concreto foi fixado com a utilização de um Funil V, em madeira, com apoio adaptado. A extremidade inferior foi fixada a uma distância aproximada de $20 \mathrm{~cm}$ do fundo da forma, conforme demonstrado na Figura 3.

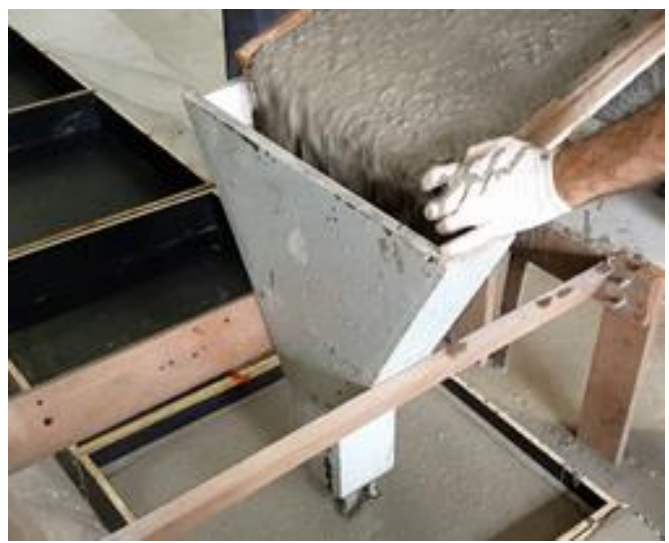

Figura 3: Funil V e apoio adaptado sendo utilizados para moldagem da placa.

A cura da placa foi feita com aplicação diária de água sobre a superfície durante sete dias. No vigésimo segundo dia após a moldagem, a placa foi marcada e cortada. Na superfície da placa foi demarcada uma borda de $2,5 \mathrm{~cm}$ e feita uma malha com 26 quadrados de $11 \mathrm{~cm}$ de lado. Cada quadrado foi identificado para que, após o corte, fosse possível identificar as suas posições segundo a moldagem inicial (Figura 4a). O corte do concreto foi feito com serra diamantada de base móvel (Figura 4b). Devido à espessura do disco de serra, a medida final das laterais ficou próxima de $10 \mathrm{~cm}$. 

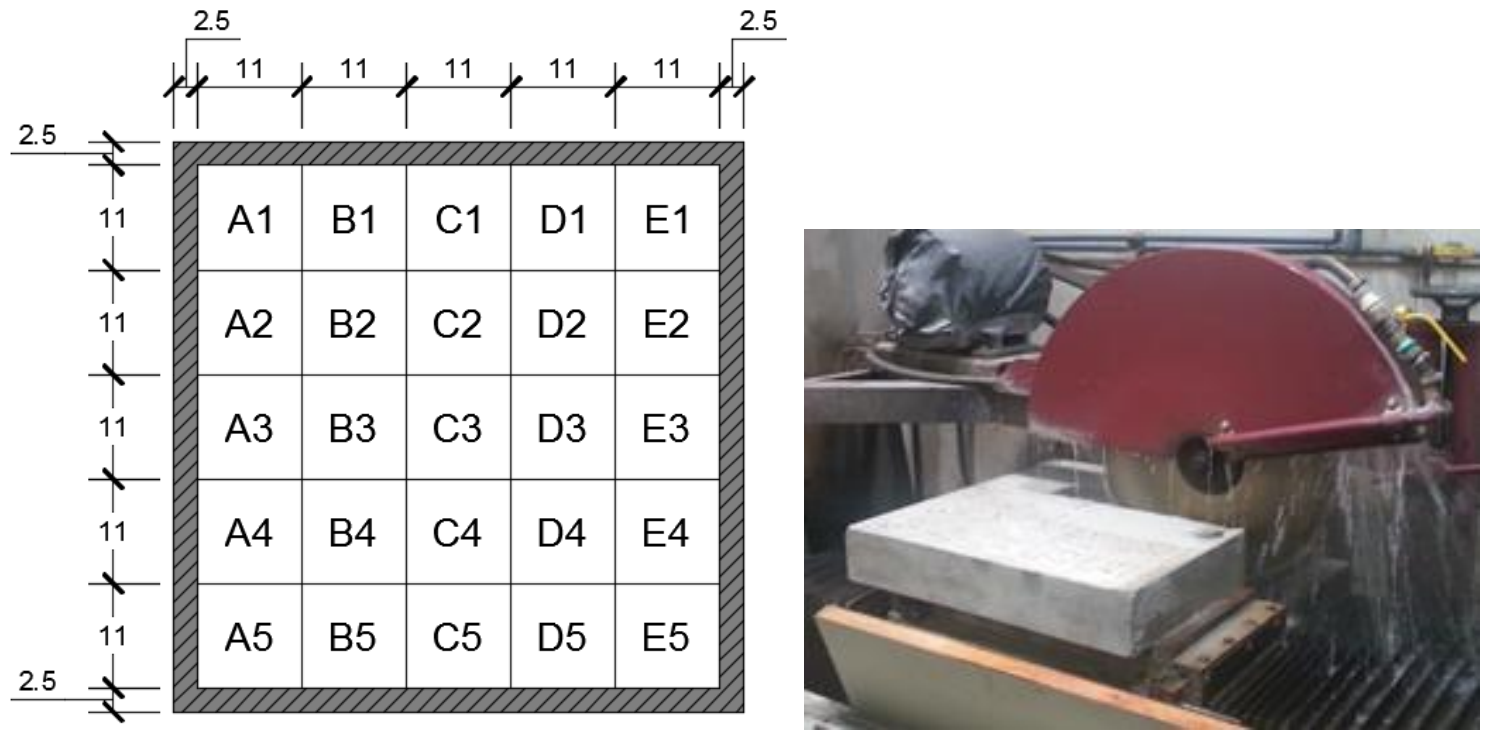

Figura 4: Marcações para corte e medidas (em centímetros) (a) e serra utilizada no corte da placa (b).

\subsection{Ensaio indutivo e calibração}

O ensaio indutivo [16] foi proposto com o objetivo de compor uma análise não-destrutiva sobre a presença e orientação das fibras de aço dentro da matriz de concreto. Esta análise consiste na leitura das alterações causadas pela presença do metal das fibras em um campo magnético gerado em uma bobina. Na concepção inicial do método, TORRENTS et al. [16] utilizavam uma bobina quadrada. Uma publicação mais recente de Cavalaro et al. [12] propôs a utilização de uma bobina circular, cujo objetivo era melhorar a homogeneidade do campo magnético gerado no interior da bobina. Essa bobina é composta por duas espirais, tipo Helmholtz, separadas em $13 \mathrm{~cm}$ e conectadas em paralelo. Cada espiral possui seção circular de $25 \mathrm{~cm}$ (medida interna) e foi produzida com 1200 voltas de fio de cobre esmaltado de $0,3 \mathrm{~mm}$ de diâmetro.

O procedimento experimental realizado neste estudo utilizou a bobina circular, ligada a um medidor LCR Agilent 4263B. O equipamento foi ajustado para frequência de $1 \mathrm{kHz}$ e tensão de $1000 \mathrm{mV}$, com medida da indutância em série (Ls). Um valor médio registrado para 20 medidas de indutância foi registrado, evitando a necessidade de repetição da leitura. A Figura 5 ilustra o equipamento e bobina utilizados.

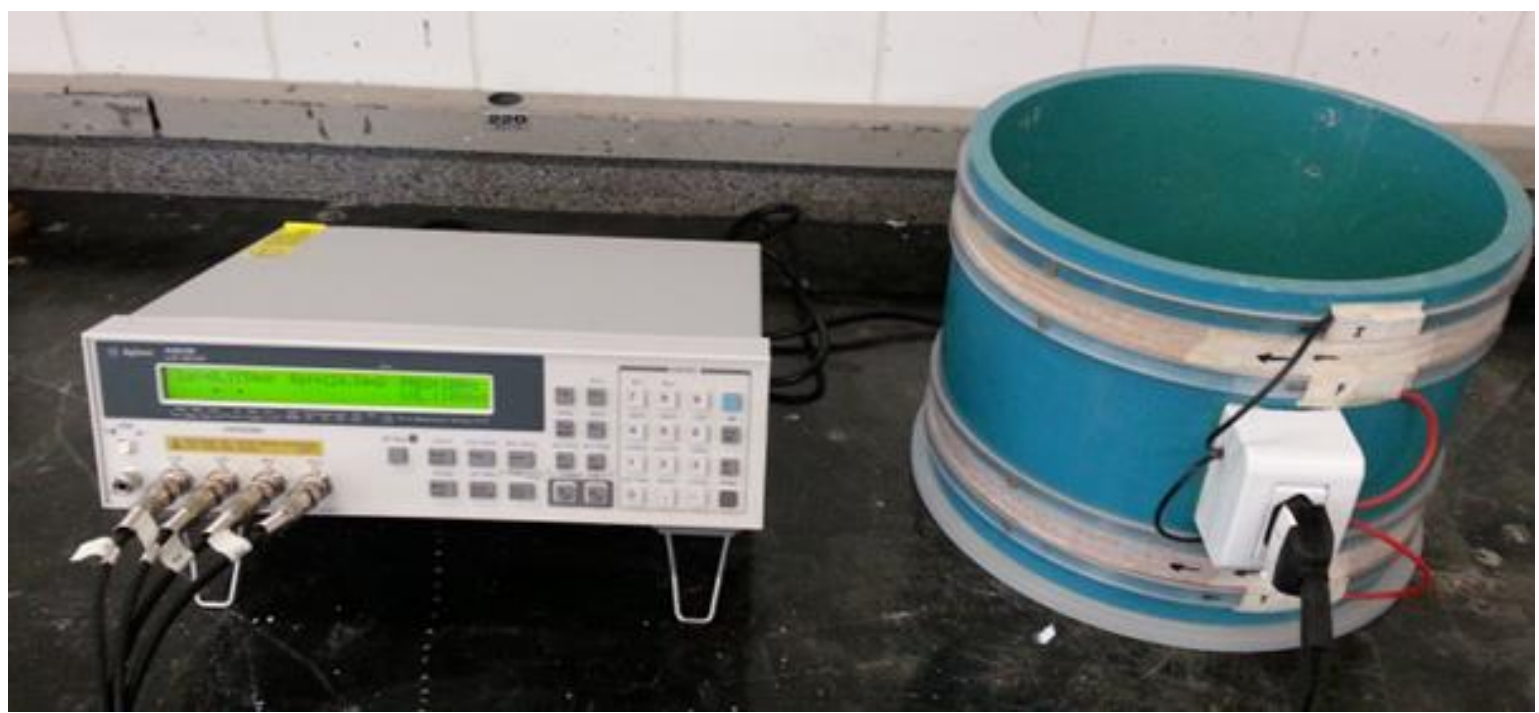

Figura 5: Bobina e leitor utilizados no ensaio indutivo.

Para a realização do ensaio, cada cubo foi colocado na bobina em três posições distintas, alinhando os três diferentes eixos em paralelo ao campo eletromagnético. Convencionou-se um sistema de eixos padrão para os cubos utilizados, o valor exibido no leitor foi aquisitado para cada posição, como apontado na Figura 6. 

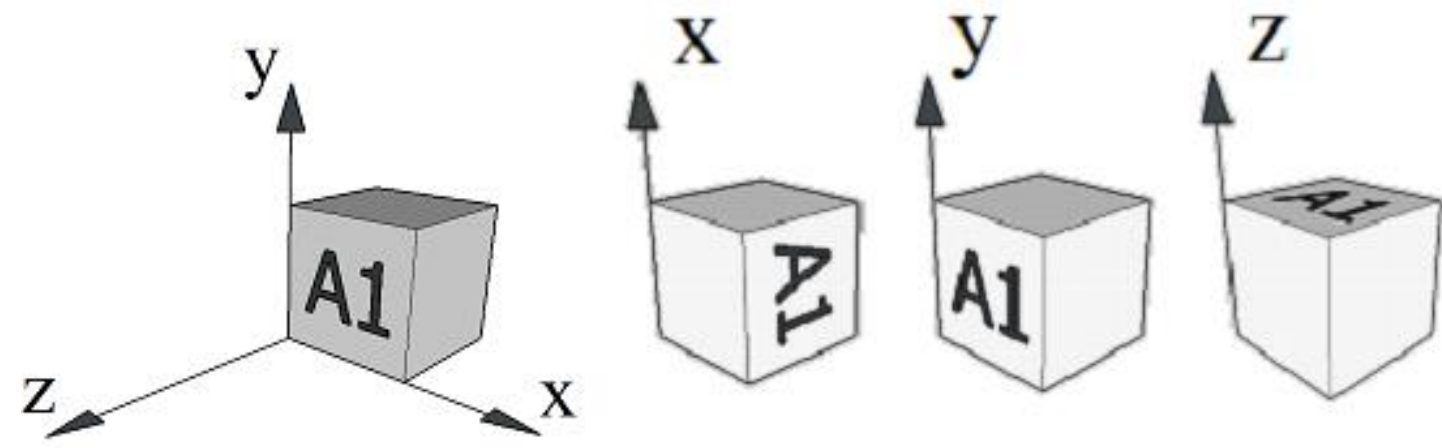

Figura 6: Sistema de eixos e posicionamento de cada eixo na bobina.

Para interpretação dos dados, é necessária a realização de uma etapa prévia de calibração. Nesta etapa, foi utilizado um cubo de material não metálico (poliestireno expandido) e de mesma conformação geométrica que os corpos de prova que foram avaliados no estudo (Figura 7). Em seguida, uma massa conhecida do mesmo tipo de fibra utilizada no estudo experimental foi distribuída no corpo de prova de calibração, que é submetido à leitura da mesma forma que os cubos de concreto que configuram os testemunhos extraídos da placa.

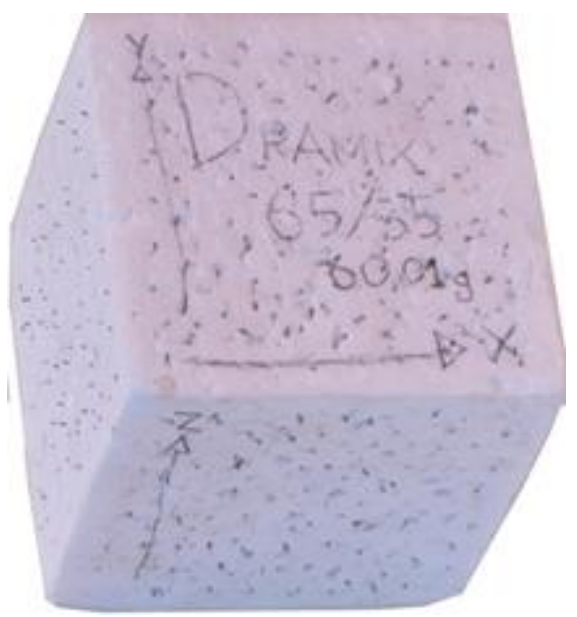

Figura 7: Exemplo de cubo de poliestireno expandido utilizado na calibração do ensaio indutivo.

As variações de indutância medidas em cada eixo $(\Delta \mathrm{Li})$ devem ser somadas, resultando a indutância total $(\Delta \mathrm{L})$ conforme equação 1 .

$$
\Delta L=\Delta L_{z}+\Delta L_{x}+\Delta L_{y}
$$

A calibração do ensaio indutivo consiste em relacionar a massa de fibras com o valor do somatório de indutância em uma regressão linear [12]. O coeficiente angular da reta obtida é a constante de relação entre a quantidade de fibras $\left(\mathrm{C}_{\mathrm{f}}\right)$ e a respectiva variação de indutância $(\Delta \mathrm{L})$, conforme a equação 2 :

$$
C_{f}=\omega . \Delta L
$$

Onde:

$\omega$ é a constante obtida pelo coeficiente angular da reta; $\mathrm{C}_{\mathrm{f}}$ é a quantidade de fibra em gramas.

O resultado da calibração da massa da fibra presente no corpo de prova está representado na Tabela 3. Com o valor de massa e a soma das leituras, foi elaborada a reta de calibração (Figura 8), da qual foi extraído o coeficiente angular, utilizado como constante para relacionar a soma das leituras com a massa de fibras. 
Tabela 3: Resultados de indutância em mH obtidos para a calibração do ensaio indutivo para a fibra Dramix 65/35 BG.

\begin{tabular}{c|c|c|c|c}
\hline MASSA $(\mathbf{g})$ & $\mathbf{\Delta L}_{\mathbf{z}}$ & $\mathbf{\Delta L}_{\mathbf{x}}$ & $\mathbf{\Delta L}_{\boldsymbol{Y}}$ & $\mathbf{\Delta} \mathbf{L}$ \\
\hline 20,03 & 0,624 & 0,733 & 0,604 & 1,961 \\
\hline 40,01 & 1,191 & 1,315 & 1,304 & 3,811 \\
\hline 60,01 & 1,837 & 1,862 & 1,852 & 5,550 \\
\hline
\end{tabular}

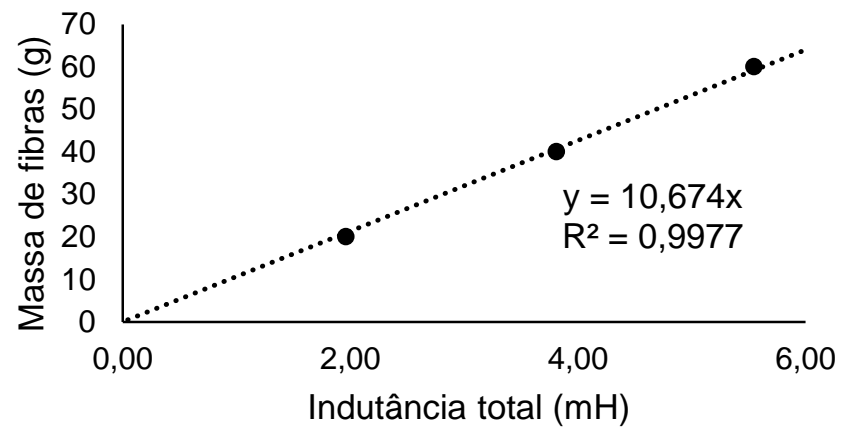

Figura 8: Reta de calibração da fibra Dramix 65/35 BG.

Para o cálculo da contribuição das fibras em determinada direção deve-se incialmente calcular o número de orientação médio $\left(n_{i}\right)$ dado por [12]:

$$
n_{i}=1,03 \cdot \sqrt{\frac{\Delta L_{i} \cdot(1+2 \cdot \gamma)-\Delta L \cdot \gamma}{\Delta L \cdot(1-\gamma)}}-0,1
$$

De posse do número de orientação $\left(n_{i}\right)$ e assumindo um valor de $\gamma$ igual a 0,03 (adotado em função do fator de forma da fibra utilizada neste estudo ser semelhante ao utilizado por Cavalaro et al. [12]) calculou-se a contribuição média real das fibras em cada um dos eixos $\left(C_{i}\right)$ através da equação 4 [12-13].

$$
C_{i}=\frac{n_{i}}{\sum_{i=X, Y, Z} n_{i}}
$$

\section{RESULTADOS}

\subsection{Concentração de fibras}

Após a calibração do método indutivo e medidas as variações de indutância nas diferentes direções, foi calculada a massa de fibra presente em cada cubo analisado, através da equação 2 . Com essa determinação e dados do volume de cada cubo foi calculado o teor de fibras em $\mathrm{kg} / \mathrm{m}^{3}$. Esse resultado é apresentado na Tabela 4, ressaltando que o ponto de concretagem da placa estava localizado sobre o cubo C3. A Tabela 5 exibe os parâmetros de caracterização da distribuição de valores obtidos na amostra de cubos extraídos da placa.

Tabela 4: Teor de fibras nos cubos $\left(\mathrm{kg} / \mathrm{m}^{3}\right)$.

\begin{tabular}{c|c|c|c|c|c}
\hline LOCALIZAÇÃO & A & B & C & D & E \\
\hline 1 & 17,4 & 22,1 & 22,5 & 18,9 & 17,5 \\
\hline 2 & 23,5 & 24,8 & 27,3 & 24,2 & 21,7 \\
\hline 3 & 26,3 & 31,0 & 27,6 & 27,5 & 23,9 \\
\hline 4 & 21,8 & 22,5 & 20,9 & 21,2 & 19,2 \\
\hline 5 & 14,2 & 17,2 & 19,8 & 16,8 & 13,8 \\
\hline
\end{tabular}


Tabela 5: Parâmetros de caracterização da amostra de concentração de fibras.

\begin{tabular}{l|c}
\hline CONCENTRAÇÃO DE FIBRAS & VALOR $\left(\mathbf{k g} / \mathbf{m}^{\mathbf{3}}\right)$ \\
\hline No ponto de moldagem & 27,62 \\
\hline Máxima & 31,05 \\
\hline Mínima & 13,80 \\
\hline Média & 21,75 \\
\hline Desvio Padrão & 4,33 \\
\hline Variância & $19,9 \%$ \\
\hline
\end{tabular}

Para melhor visualização dos resultados, os valores de teor de fibras foram colocados num gráfico 3D de superfície que é apresentado na Figura 9. Pode-se observar por este gráfico que a distribuição das fibras na placa apresentou uma maior concentração na porção central da mesma, coincidindo com o ponto de lançamento do material. Os valores de consumo de fibra nessa posição foram ligeiramente maiores que nas extremidades da placa. Isto sugere claramente uma segregação associada a uma maior mobilidade da mistura em relação à fibra. Dessa forma, o deslocamento mais lento da fibra fez com que ela se concentre mais na região próxima do ponto de lançamento.

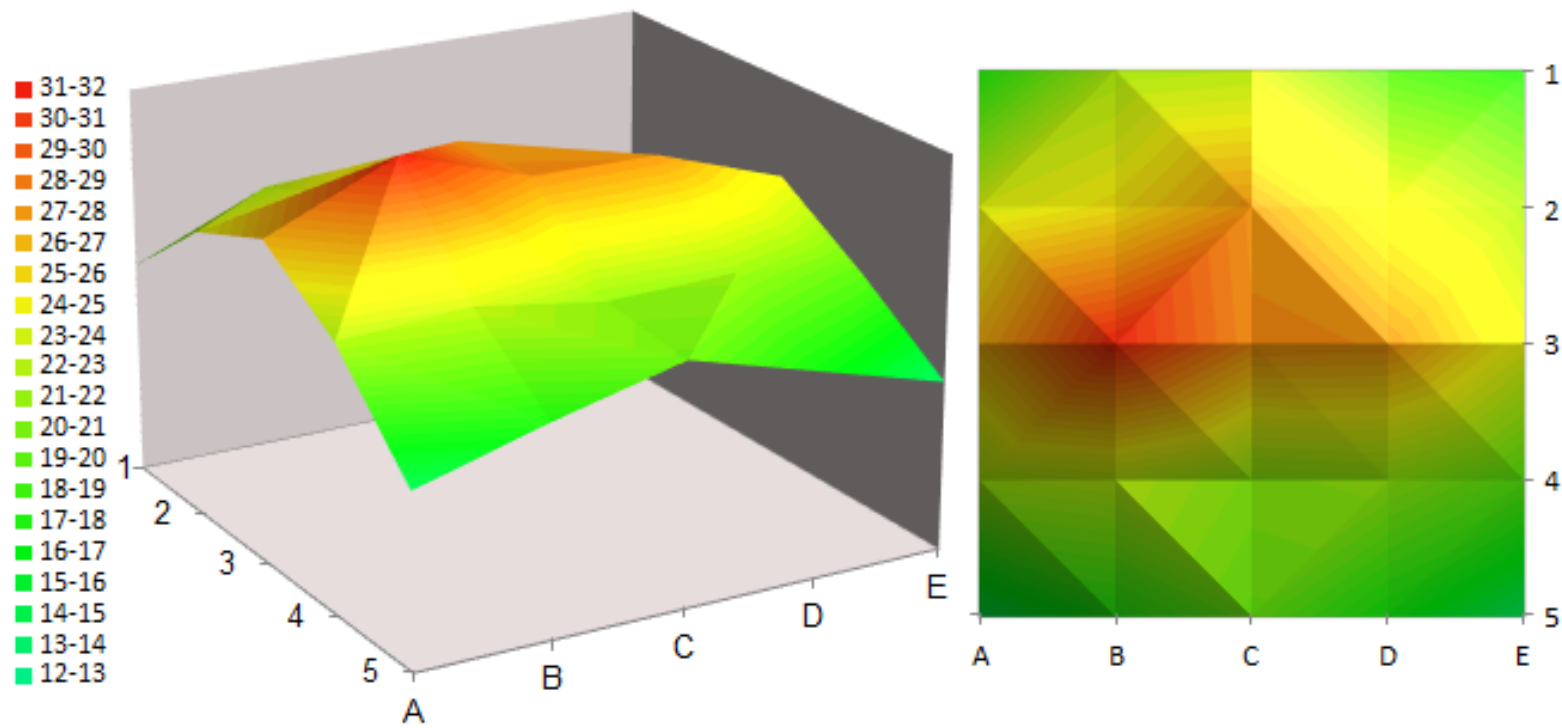

Figura 9: Concentração de fibras $\left(\mathrm{kg} / \mathrm{m}^{3}\right)$ na placa.

\subsection{Orientação das fibras}

O gráfico da Figura 10 traz a relação entre a contribuição de fibras medida no eixo z (eixo vertical) e a distância do ponto de moldagem até o centro de cada cubo. O resultado indica a tendência de contribuição cada vez menor no eixo z à medida que se distancia do ponto de moldagem. 


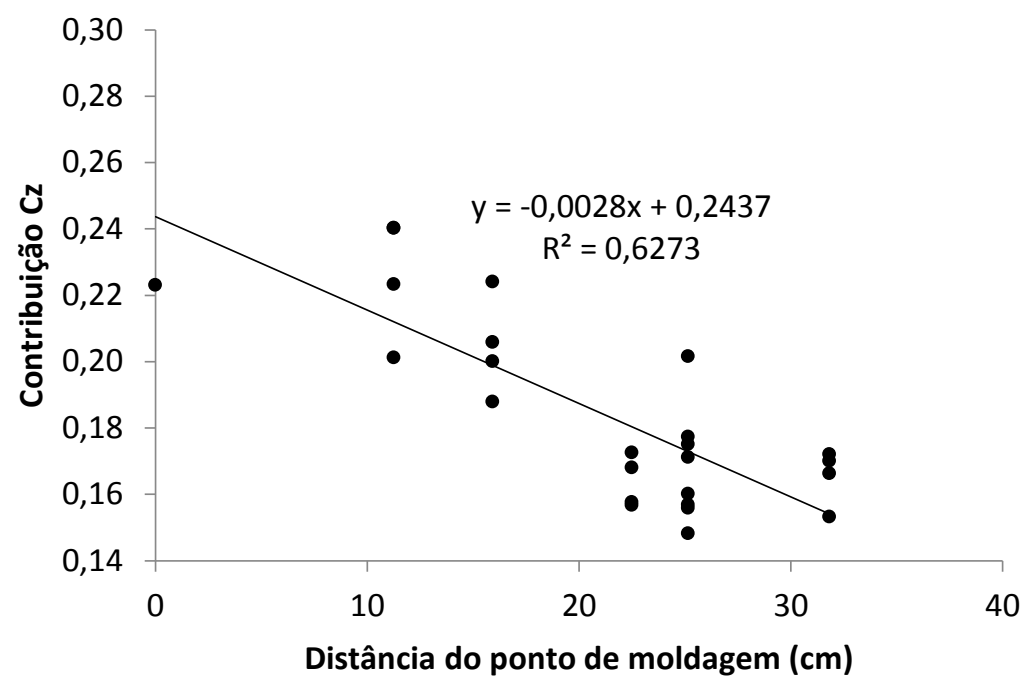

Figura 10: Relação entre distância de moldagem e fibras alinhadas com o eixo Z.

$\mathrm{Na}$ análise dos eixos no plano horizontal, foi elaborado um mapa de orientação da placa. A Figura 11 ilustra a contagem das fibras nos eixos x e y de cada cubo. É possível perceber que a contribuição das fibras nos eixos varia em função do fluxo de concreto, partindo do ponto de moldagem (destacado em cinza).

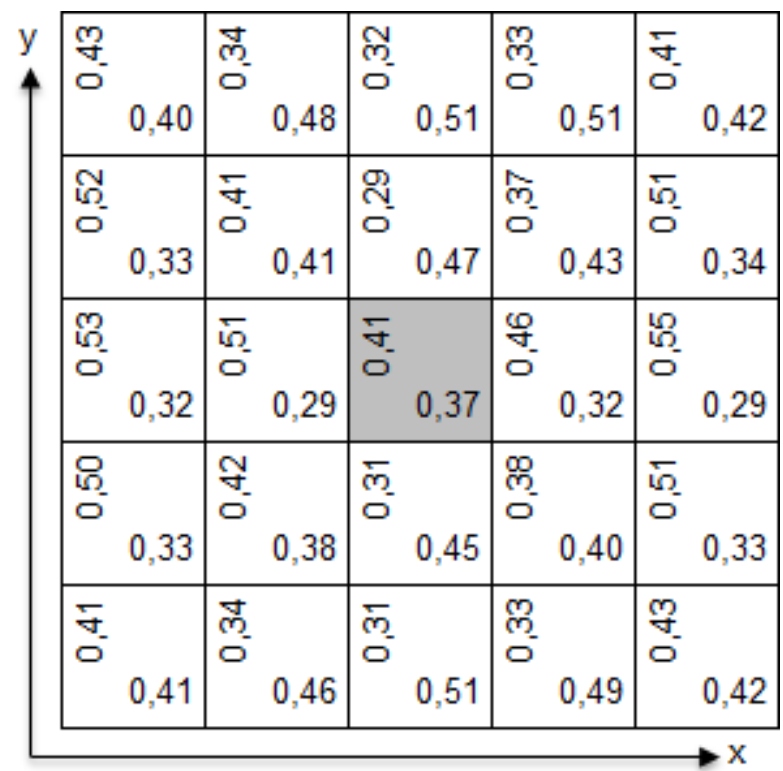

Figura 11: Contribuições nos eixos x e y

Nomeou-se, então, um sistema de eixos partindo do ponto de moldagem, a fim de realizar a análise da influência do fluxo sobre a orientação de fibras. O sistema foi dividido em quatro eixos, nomeados como ortogonais 1 e 2 e como diagonais 1 e 2, e estão indicados na Figura 12. Esta divisão permitiu a realização da análise entre os eixos equivalentes. 


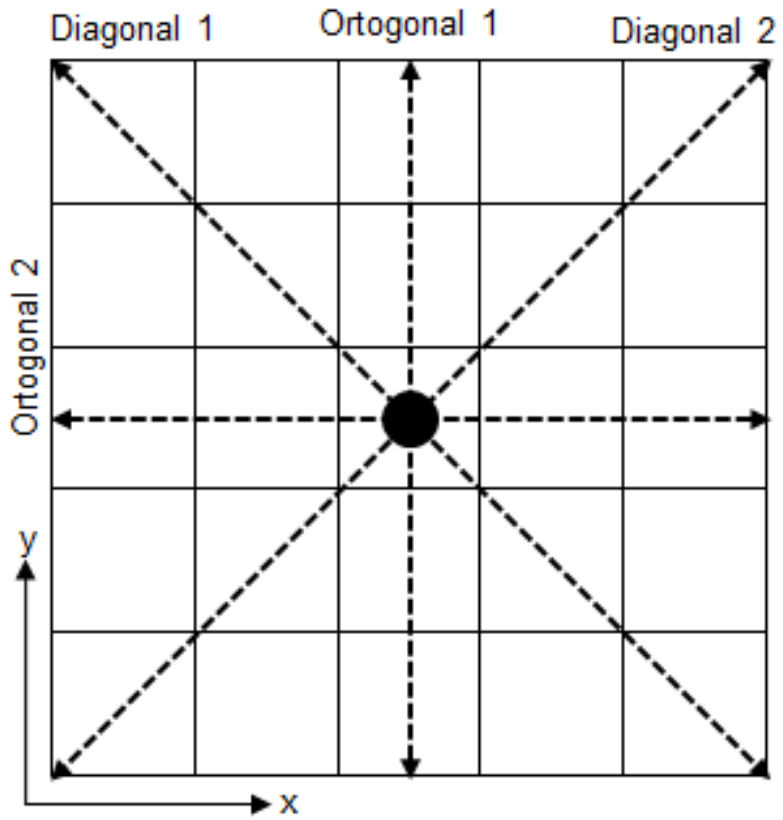

Figura 12: Eixos nomeados para análise do fluxo

Para que, na análise feita, fossem comparadas as fibras orientadas na mesma posição em relação ao ponto de lançamento, a nomeação nos eixos ortogonais foi dividida entre paralela ou transversal ao sentido do fluxo. Já nos eixos diagonais, por estarem sempre alinhados com a direção do fluxo, não foi necessária alteração do nome. A Figura 13 ilustra a classificação utilizada para avaliação dos eixos.
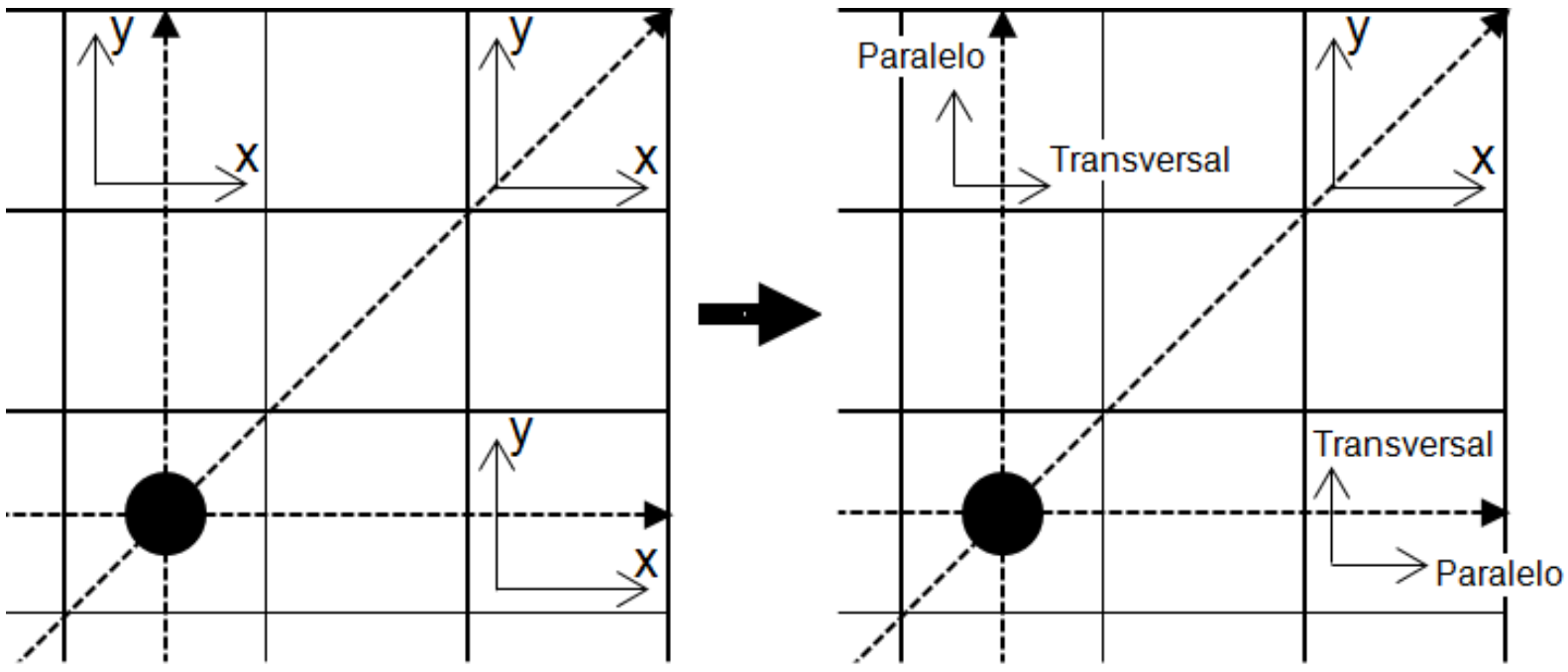

Figura 13: Exemplo de nomeação dos eixos de leitura do ensaio indutivo: antes (a) e depois (b)

A contagem de fibras foi, então, analisada para cada corpo de prova. O cubo sob o ponto de lançamento do concreto foi numerado como 0 , e os demais cubos foram numerados de $-2 \mathrm{a}+2$.

No resultado, mostrado na Figura 14, é possível observar que os dois eixos ortogonais apresentam comportamento semelhante, pois, à medida que o concreto flui do centro para as laterais da forma, as fibras tendem a reduzir a orientação no sentido paralelo à linha de fluxo enquanto aumentam a orientação no sentido transversal. Os eixos diagonais também apresentam comportamentos semelhantes entre si, pois as fibras não apresentaram mudanças significativas na orientação medida, apresentando valores quase iguais nas direções x e $y$, o que indica que as fibras tendem a ficar igualmente divididas entre os eixos $\mathrm{x}$ e y nos cubos ao longo do eixo de fluxo diagonal. 


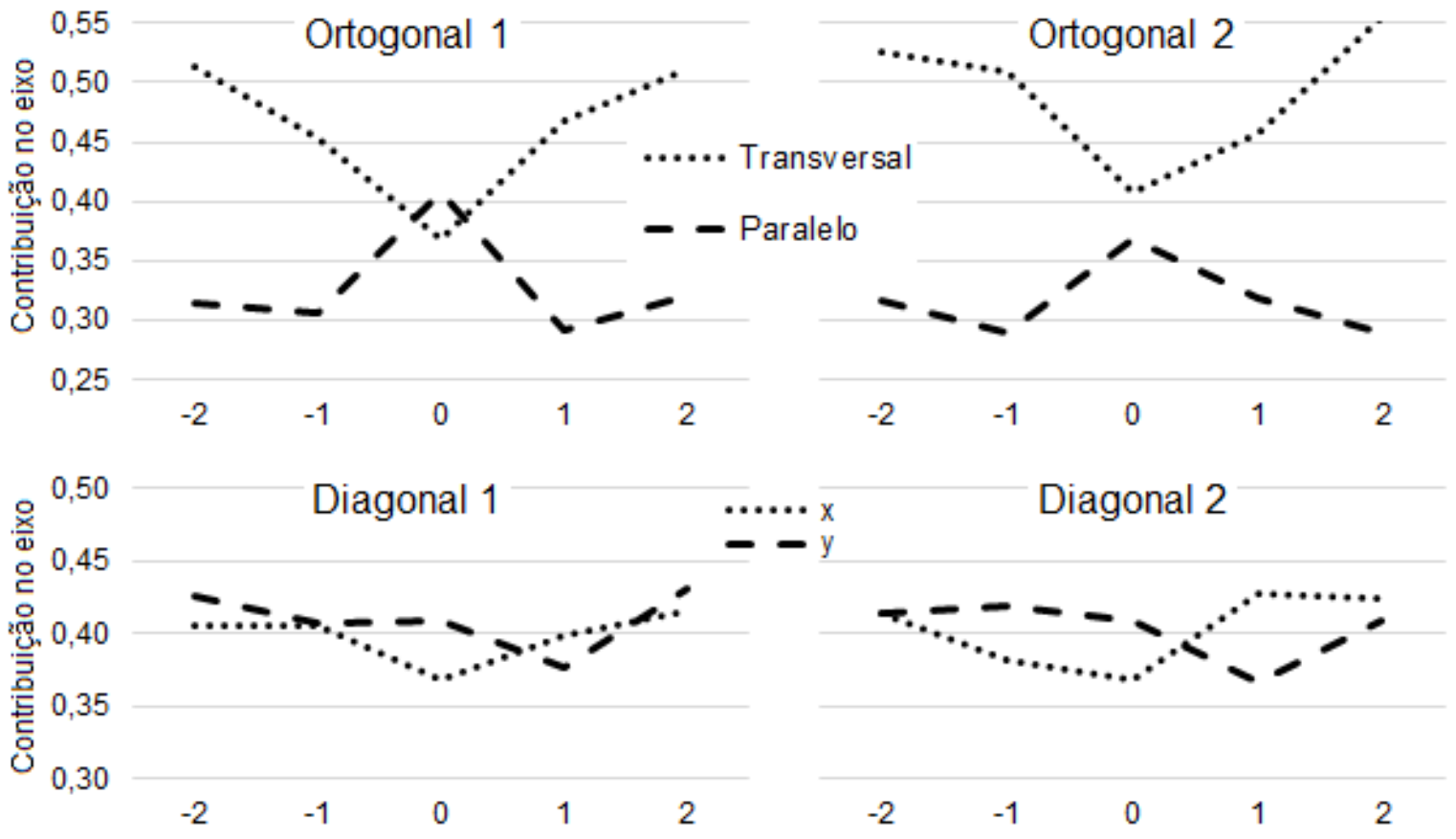

Figura 14: Análise do direcionamento das fibras ao longo do fluxo

De modo a possibilitar uma maneira adequada de avaliar a orientação preferencial das fibras, foi calculada uma proporção relativa entre os eixos x e y, analisando-se apenas a variação da direção das fibras no plano horizontal.

No cálculo da proporção, quando x e y são aproximadamente iguais, a razão entre os valores é próxima de 0,5. Considerando-se que as fibras estariam igualmente alinhadas com ambos os eixos, este valor foi associado ao ângulo de $45^{\circ}$ (Figura 15). De forma análoga, a proporção mais desigual entre eixos foi associada aos ângulos de $90^{\circ}$ e $0^{\circ}$, pois indicam a condição do alinhamento hipotético das fibras em uma direção.

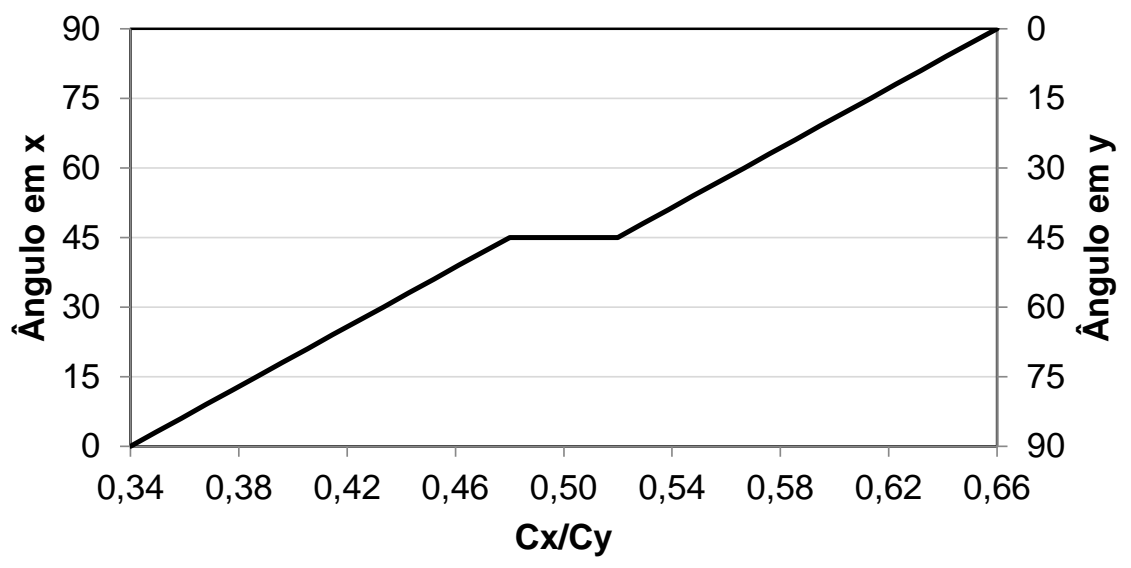

Figura 15: Gráfico de relação entre razão $\mathrm{Cx} / \mathrm{Cy}$ e ângulo adotado

Uma vez atribuídos os ângulos de orientação preferencial, a representação dos ângulos com linhas posicionadas no centro de cada cubo foi feita, com a inclinação mais provável correspondente. O resultado, apresentado na Figura 16, indica a tendência de direcionamento das fibras presentes no plano horizontal daquele cubo, não levando em consideração a inclinação no eixo z. 


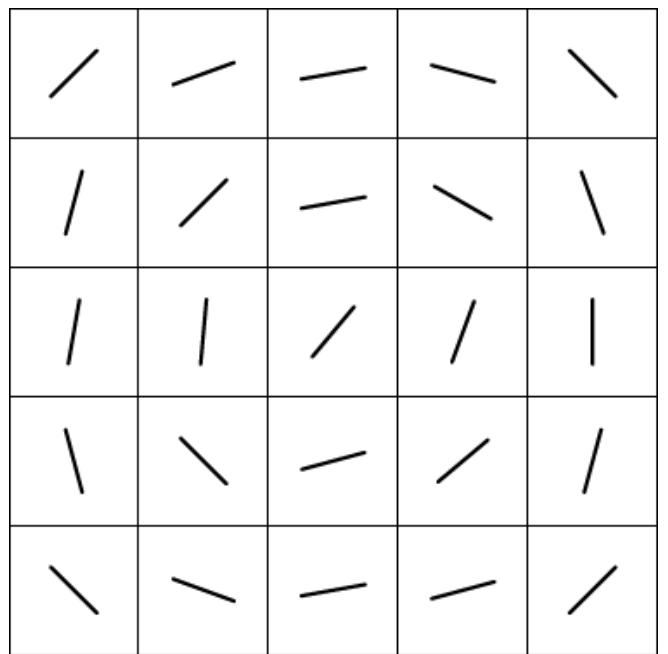

Figura 16: Angulações calculadas para tendência de alinhamento das fibras nos eixos x e y.

A partir da tendência individual do alinhamento hipotético das fibras de cada cubo, é possível traçar linhas de fluxo que descrevem a orientação preferencial nas placas partindo do ponto de lançamento do concreto (Figura 17).

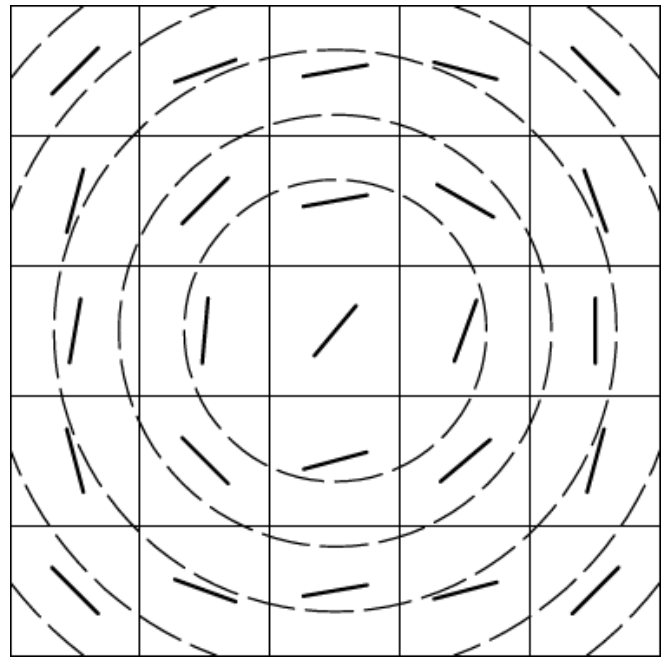

Figura 17: Linhas de fluxo traçadas com base no alinhamento calculado das fibras

Vale ressaltar que esta metodologia não define precisamente o ângulo em que as fibras estão no concreto, apenas indica a tendência de alinhamento preferencial nos planos x e y em relação à posição de moldagem.

\section{DISCUSSÃO}

\subsection{Concentração de fibras}

O ensaio indutivo permitiu a realização de medidas com informações acerca da distribuição de concentração de fibras nas placas a partir dos cubos de concreto. Assim, foi possível observar que a concentração média de fibras mostrou-se acima do valor previsto de $20 \mathrm{~kg} / \mathrm{m}^{3}$. Isto ocorre porque a dosagem nos caminhões betoneira foi feita em massa com estimativa de volume do concreto do caminhão betoneira e variações podem ocorrer no processo, como variações no teor de ar aprisionado por exemplo. Esta diferença no teor de fibras previsto, no entanto, pode afetar o comportamento do compósito e, consequentemente, do elemento estrutural com ele produzido. Por essa razão, é recomendável que haja uma verificação do teor de fibra real dentro dos programas de controle de qualidade do CRF.

O ensaio de espalhamento apresentou diâmetros acima do recomendado ela EFNARC [15], no entanto, 
estes valores não são restritivos para a caracterização do concreto. Além disso, foram estabelecidos para avaliar a fluidez no uso de concreto sem reforço de fibras. Na medida do $\mathrm{T}_{500}$, um curto tempo de espalhamento está associado à condição de baixa viscosidade no concreto, como sugere a EFNARC [15]. Este aspecto pode ter sido responsável pela distribuição irregular de fibras, uma vez que o concreto não teria sido capaz de exercer força de arrasto suficiente para espalhar a mesma quantidade de fibras até os cantos da forma. Tal tipo de condição exigiria ajustes na dosagem do material de modo a corrigir esta limitação. Isto resultou numa maior concentração de fibras no meio da placa, que pode ser algo positivo se esta região do elemento for a mais solicitada, como acontece com uma carga concentrada no centro de uma laje. Como observado em ALFERES FILHO [17], quando a moldagem é feita pela borda a concentração de fibras também é maior nesta região em relação ao centro e ao extremo oposto à posição de lançamento do concreto.

\subsection{Orientação de fibras}

$\mathrm{Na}$ análise de orientação das fibras, é possível notar uma tendência de redução da verticalidade das fibras causada pelo fluxo (Figura 10). À medida que as fibras se afastam do centro, há uma orientação mais próxima do plano horizontal, isto é explicado pelo fato que a orientação vertical nas fibras está relacionada à influência do fluxo vertical ocorrido no funil durante o lançamento. Este resultado é muito próximo do padrão observado no estudo de DEEB et al. [18]

A partir da contribuição das fibras nos eixos x e y, foi possível atribuir a tendência predominante de alinhamento das fibras em cada região da placa. Com o traçado das linhas de fluxo, observou-se que as fibras apresentam orientação preferencial em posição transversal à linha de fluxo do concreto. E esta orientação se acentua nos cubos mais distantes do ponto de moldagem, devido ao maior tempo sob influência do fluxo. Estes resultados se assemelham à proposição de ABRISHAMBAF et al. [5] feita com base em análise de imagens (Figura 18a), aos resultados obtidos por PONIKIEWSKI et al. [19] com análise feita por tomografia computadorizada, e ao espalhamento obtido por BOULEKBACHE et al. [20] com fluido translúcido incorporado com fibras de aço (Figura 18b).
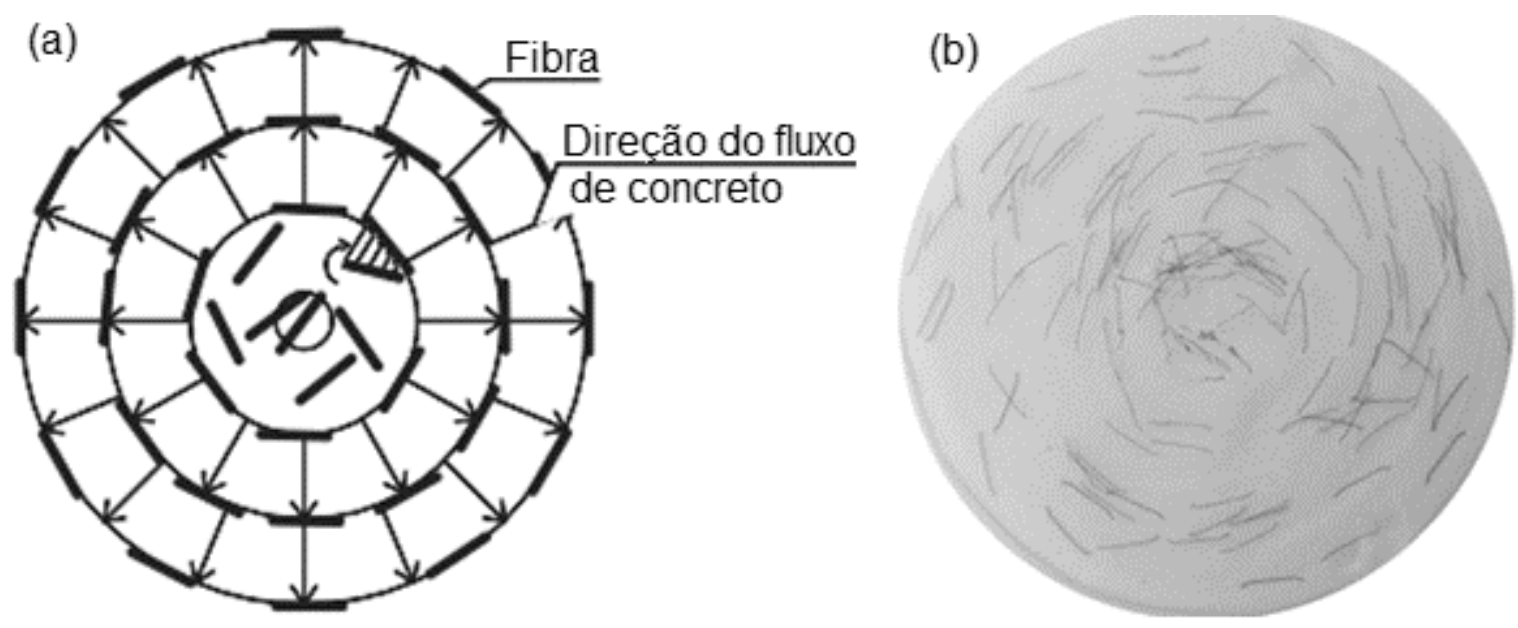

Figura 18: Perfil de alinhamento obtido em outros estudos

Fonte: ABRISHAMBAF et al. [5] (a) e BOULEKBACHE et al [20] (b)

\section{CONCLUSÕES}

Este estudo demonstrou a capacidade do ensaio indutivo no mapeamento das condições de distribuição das fibras de aço em elementos planos de concreto autoadensável. Isto ocorreu tanto em termos de concentração ou conteúdo de fibras como de orientação preferencial. Dessa forma, foi possível detectar que a distribuição da fibra não ocorre de maneira uniforme durante o lançamento do concreto na fôrma, tanto em termos de conteúdo como de orientação.

As condições de moldagem resultaram em concentrações mais elevadas de fibras na porção central da placa onde o concreto foi vertido, possivelmente ocasionado por uma baixa viscosidade do concreto. Além disso, na análise de orientação nos três eixos, o distanciamento do ponto de concretagem induziu a orientação das fibras no plano horizontal. O fluxo de concreto durante a moldagem da placa também causou direções preferenciais de orientação das fibras no sentido transversal às linhas de fluxo. 
Estes resultados apontam que não se pode ignorar a importância do controle de execução de elementos estruturais executados com CRF de modo a evitar a ocorrência de orientações preferenciais que prejudiquem o comportamento do elemento, uma vez que a orientação das fibras pode gerar diferentes níveis de capacidade de reforço em função do padrão de fissuração estabelecido no elemento estrutural.

Pode-se afirmar que o ensaio indutivo pode contribuir para o controle tecnológico do CRF no controle da variação do teor de fibras efetivamente incorporado ao concreto. Como se observou neste estudo, há possibilidade de o teor efetivo variar em relação ao teórico e a falta de controle neste aspecto pode implicar em prejuízo ao comportamento mecânico do CRF no caso do valor efetivo ser inferior ao teórico. O ensaio indutivo não é destrutivo em relação ao corpo de prova e pode ser associado a outros métodos de ensaio corriqueiros, como o de determinação da resistência à compressão e absorção de água, de modo a aumentar o rigor do processo de controle de qualidade. Porém, é necessário a extração de testemunhos para que possa ser identificada a distribuição e concentração das fibras no elemento estrutural. Outros métodos baseados nas propriedades magnéticas das fibras de aço vêm sendo estudados como alternativa para o controle de campo sem a necessidade de extração [21,22].

\section{AGRADECIMENTOS}

Os autores agradecem à FUSP e à Engemix pelo apoio para realização desta pesquisa.

\section{BIBLIOGRÁFIA}

[1] FÉDÉRATION INTERNATIONALE DU BÉTON - FIB. Fib Model Code for Concrete Structures 2010. Switzerland, 2013. 402p.

[2] DI PRISCO, M., PLIZZARI, G., VANDEWALLE, L. "Fibre reinforced concrete: new design perspectives", Materials and Structures, v. 42, pp. 1261-1281, Set. 2009.

[3] FIGUEIREDO, A. D. Concreto reforçado com fibras. Tese (Livre-Docência), Escola Politécnica/USP, São Paulo, SP, 2011.

[4] FERRARA, L., et al., "A comprehensive methodology to test the performance of Steel Fibre Reinforced Self-Compacting Concrete (SFR-SCC)", Construction and Building Materials, v. 37, pp. 406-424, Dez. 2012.

[5] ABRISHAMBAF, A., BARROS, J. A. O., CUNHA, V. M. C. F. "Relation between fibre distribution and post-cracking behaviour in steel fibre reinforced self-compacting concrete panels", Cement and Concrete Research, v. 51, pp. 57-66, Set. 2013.

[6] ABUKHASHABA, M. I., MOSTAFA, M. A., ADAM, I. A. "Behavior of self-compacting fiber reinforced concrete containing cement kiln dust", Alexandria Engineering Journal, v. 53, n. 2, pp. 341-354, Jun. 2014.

[7] AKCAY, B., TASDEMIR, M. A, "Mechanical behaviour and fibre dispersion of hybrid steel fibre reinforced self-compacting concrete", Construction and Building Materials, v. 28, n. 1, pp. 287-293, Mar. 2012.

[8] ZERBINO, R., et al., "On the orientation of fibres in structural members fabricated with self-compacting fibre reinforced concrete”, Cement and Concrete Composites, v. 34, n. 2, pp. 191-200, Fev. 2012.

[9] BLANCO, A., et al., "Assessment of the fibre orientation factor in SFRC slabs", Composites Part B: Engineering, v. 68, pp. 343-354. 2015.

[10] LATASTE, J. F., BEHLOUL, M., BREYSSE, D. "Characterisation of fibres distribution in a steel fibre reinforced concrete with electrical resistivity measurements", NDT \& E International, v. 41, n. 8, pp. 638647, Dez. 2008.

[11] AL-MATTARNEH, H, "Electromagnetic quality control of steel fiber concrete", Construction and Building Materials, v. 73, pp. 350-356, Dez. 2014.

[12] CAVALARO, S. H. P., et al., "Improved assessment of fibre content and orientation with inductive method in SFRC”, Materials and Structures, v. 48, pp. 1859-1873, 2015.

[13] LÓPEZ, R.D., et al., Assessment of Fibre Content and Orientation in SFRC with the Inductive Method. Part 1: Theoretical Basis of the Method and Study of the Influence of the Type of Coil and Temperature on its Accuracy. E-Journal of Nondestructive Testing and Ultrasonics, v. 20, p. 18383, 2015. 
[14] SILVA, C. L., et al., "Assessment of Fibre Content and Orientation in SFRC with the Inductive Method. Part 2: Application for the Quality Control of Sprayed Concrete", E-Journal of Nondestructive Testing and Ultrasonics, v. 20, p. 18384, 2015.

[15] EFNARC. The European Guidelines for Self-Compacting Concrete; specification, production and use. European Federation of Producers and Applicators of Specialist Products for Structures (EFNARC), 2005.

[16] TORRENTS, J. M., et al., "Inductive method for assessing the amount and orientation of steel fibers in concrete", Materials and Structures, v. 45, n. 10, pp. 1577-1592, 24 Abr. 2012.

[17] ALFERES FILHO, R. Avaliação da influência do direcionamento de fibras de aço no comportamento mecânico de concreto autoadensável aplicado em elementos planos. Dissertação de M.sc., Escola Politécnica, Universidade de São Paulo, São Paulo, 2016.

[18] DEEB, R., KARIHALOO, B. L., KULASEGARAM, S. "Reorientation of short steel fibres during the flow of self-compacting concrete mix and determination of the fibre orientation factor", Cement and Concrete Research, v. 56, pp. 112-120, Fev. 2014.

[19] PONIKIEWSKI, T., et al., "Determination of steel fibres distribution in self-compacting concrete beams using X-ray computed tomography”, Archives of Civil and Mechanical Engineering, v. 15, n. 2, pp. 558-568, Fev. 2015.

[20] BOULEKBACHE, B., et al., "Flowability of fibre-reinforced concrete and its effect on the mechanical properties of the material”, Construction and Building Materials, v. 24, n. 9, pp. 1664-1671, Set. 2010.

[21] FERRARA, L., FAIFER, M., TOSCANI, S. "A magnetic method for non-destructive monitoring of fiber dispersion and orientation in steel fiber reinforced cementitious composites-part 1: Method calibration", Materials and Structures, v. 45, n. 4, pp. 575-589. Abr. 2012.

[22] NUNES, S., PIMENTEL, M., CARVALHO, A., "Non-destructive assessment of fibre content and orientation in UHPFRC layers based on a magnetic method", Cement and Concrete Composites, v. 72, pp. 6679. Set. 2016.

\section{ORCID}

Ricardo Alferes

Renata Monte

Antonio Figueiredo https://orcid.org/0000-0003-0807-4875

https://orcid.org/0000-0003-3555-4655

https://orcid.org/0000-0003-4658-3355 